\title{
Plaquette Ising models, degeneracy and scaling
}

\author{
Desmond A. Johnston $^{1, a}$, Marco Mueller ${ }^{2, b}$, and Wolfhard Janke ${ }^{2, c}$ \\ 1 Department of Mathematics, School of Mathematical and Computer Sciences, \\ Heriot-Watt University, Riccarton, Edinburgh EH14 4AS, Scotland \\ 2 Institut für Theoretische Physik, Universität Leipzig, Postfach 100 920, \\ 04009 Leipzig, Germany
}

Received 16 October 2016 / Received in final form 30 November 2016 Published online 5 April 2017

\begin{abstract}
We review some recent investigations of the $3 d$ plaquette Ising model. This displays a strong first-order phase transition with unusual scaling properties due to the size-dependent degeneracy of the low-temperature phase. In particular, the leading scaling correction is modified from the usual inverse volume behaviour $\propto 1 / L^{3}$ to $1 / L^{2}$. The degeneracy also has implications for the magnetic order in the model which has an intermediate nature between local and global order and gives rise to novel fracton topological defects in a related quantum Hamiltonian.
\end{abstract}

\section{Introduction}

The Ising model [1] with ferromagnetic nearest-neighbour interactions serves as a paradigm for ferromagnetic phase transitions and is almost certainly the most studied single model in statistical mechanics $[2-5]$. In general, outside the field of disordered systems Ising models with multispin interactions have been less explored [6,7], both because such interactions are less common in real materials and also because in many cases such models display first-order transitions. These might be regarded as a priori less promising subjects for numerical investigation, although the general scaling theory for first-order transitions, initiated in [8-10] and developed further in [11-15] and [16-19], is now generally well-understood. In disordered systems, of course, multispin interaction Hamiltonians play an important role in the random first-order transition (RFOT) theory of spin glasses [20-22].

Here, we remain within the ambit of purely ferromagnetic couplings and review some recent work on one of the simplest multispin interaction models, a $3 d$ plaquette Ising Hamiltonian with the spins $\sigma= \pm 1$ sited at the vertices of a $3 d$ cubic lattice,

$$
H=-\frac{1}{2} \sum_{[i, j, k, l]} \sigma_{i} \sigma_{j} \sigma_{k} \sigma_{l} .
$$

\footnotetext{
a e-mail: D.A.Johnston@hw.ac.uk

b e-mail: Marco.Mueller@itp.uni-leipzig.de

c e-mail: Wolfhard.Janke@itp.uni-leipzig.de
} 
The four-spin interactions in the Hamiltonian are between spins situated on the corners of the plaquettes that comprise each cube.

The purely plaquette Hamiltonian of Equation (1) can be thought of as the limiting case, for $\kappa \rightarrow 0$, of a one-parameter family of $3 d$ gonihedric Ising Hamiltonians [23-31]. These contain in general nearest-neighbour $\langle i, j\rangle$, next-to-nearest-neighbour $\langle\langle i, j\rangle\rangle$ and plaquette $[i, j, k, l]$ interactions,

$$
H^{\kappa}=-2 \kappa \sum_{\langle i, j\rangle} \sigma_{i}, \sigma_{j}+\frac{\kappa}{2} \sum_{\langle\langle i, j\rangle\rangle} \sigma_{i}, \sigma_{j}-\frac{1-\kappa}{2} \sum_{[i, j, k, l]} \sigma_{i} \sigma_{j} \sigma_{k} \sigma_{l},
$$

where the ratios of the various couplings have been fine-tuned to eliminate the bare surface tension, which in the case of the standard nearest-neighbour Ising Hamiltonian is the only term. When regarded as a model of fluctuating surfaces described by the geometric spin cluster boundaries the intention is to weight the edge length of spin clusters rather than their boundary area [32].

For $\kappa \neq 0$ the $3 d$ Hamiltonians $H^{\kappa}$ display a continuous transition, possibly with $3 d$ Ising exponents which are masked by strong crossover effects from the nearby tricritical point $[33,34]$. The $\kappa=0$ plaquette Hamiltonian, on the other hand, has a strong first-order phase transition [35] and recent high-precision multicanonical simulations [36-39] have revealed that it displays non-standard finite-size scaling properties at this transition. In the sequel we will describe how this non-standard scaling is a consequence of a macroscopic low-temperature phase degeneracy in the $3 d$ plaquette Ising model and also discuss the implications of this degeneracy for the nature of the low-temperature phase and the definition of an order parameter [40-42]. Recent work on quantum spin versions of the plaquette model has also shown that the symmetries of the plaquette Hamiltonian and the low-temperature degeneracy are instrumental in the appearance of a novel form of topological defect, a fracton [43-48], and we sketch their appearance via a partial gauging construction using the quantum dual of the plaquette model.

\section{A curious symmetry - classical aspects}

The Hamiltonians of Equations (1) and (2) have symmetry properties which are intermediate between those of a gauge theory and a global symmetry. Consideration of how the spin configuration on a cubic lattice can be built up from the individual cubes $[32,49]$ shows that the spins on any face(s) of a single cube may be flipped at zero energy cost. Since an entire ground-state lattice configuration may be built by stacking compatibly shaded cubes this means that there is freedom to flip planes of spins at zero energy cost, see Figure 1.

When $\kappa \neq 0$ we can see that parallel non-intersecting planes of spins may be flipped at zero energy cost at zero temperature, giving a $3 \times 2^{L}$ ground-state degeneracy on an $L^{3}$ cubic lattice. When $\kappa=0$, on the other hand, the constraint on intersections is relaxed so any planes may be flipped and the ground-state degeneracy becomes $2^{3 L}$, as illustrated in Figure 2.

When $\kappa \neq 0$ the ground-state degeneracy is broken at finite temperature, as is revealed by a low-temperature expansion [50,51]. When $\kappa=0$, however, the $2^{3 L}$ degeneracy persists into the low-temperature phase. Such macroscopic ground-state degeneracy is not uncommon in other systems, what is rarer is its persistence into the low-temperature phase. The nearest-neighbour Ising antiferromagnet on a FCC lattice, for instance, also has a macroscopically degenerate ground-state in which crystal planes of spins may be flipped, but only six possible ordered phases survive from these at finite temperature $[52,53]$. 

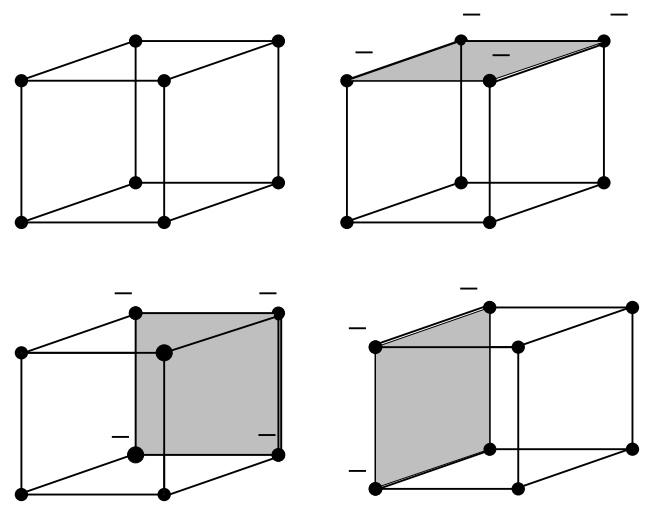

Fig. 1. Flipping the value of the Ising spins on a face of a single cube does not change its contribution to the energy at $T=0$. All of the configurations shown have the same energy.

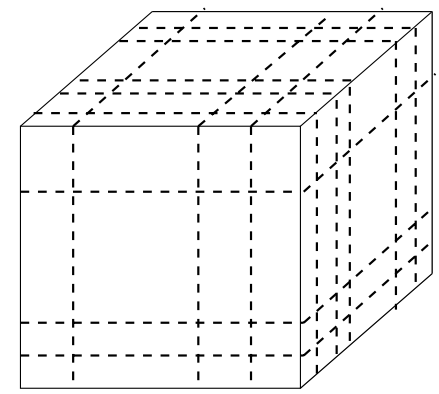

Fig. 2. A typical ground state of the $3 d$ plaquette Hamiltonian showing the edges of the planes of spins that are flipped with respect to a purely ferromagnetic ground state dotted. Since any plane of spins in any of the three possible orientations may be flipped in such a configuration, the ground-state degeneracy on an $L^{3}$ lattice is $q=2^{3 L}$.

The dual of the plaquette Hamiltonian is an anisotropically coupled Ashkin-Teller model

$$
H_{\mathrm{dual}}=-\frac{1}{2} \sum_{\langle i j\rangle} \sigma_{i} \sigma_{j}-\frac{1}{2} \sum_{\langle i k\rangle} \tau_{i} \tau_{k}-\frac{1}{2} \sum_{\langle j k\rangle} \sigma_{j} \sigma_{k} \tau_{j} \tau_{k}
$$

with two flavours of Ising spins $\sigma, \tau[54]$ and possesses the same ground-state degeneracy as the original plaquette Hamiltonian, since it is still possible to flip planes of spins at zero energy cost, see Figure 3. Although it has not been confirmed with a low-temperature expansion in the manner of the plaquette Hamiltonian, the finite-size scaling properties at the first-order transition in the dual model show that the macroscopic degeneracy persists into the low-temperature phase there too, as we discuss below.

In the rest of this review we will describe the consequences of the macroscopic low-temperature phase degeneracy in the $3 d$ plaquette Ising model (and its dual) for the finite-size scaling at the first-order phase transition and the implications for the definition of an order parameter. We then outline the role played by the spin-flip symmetry in enabling the appearance of fracton topological defects in a Hamiltonian related to the dual of the quantum version of the model. 


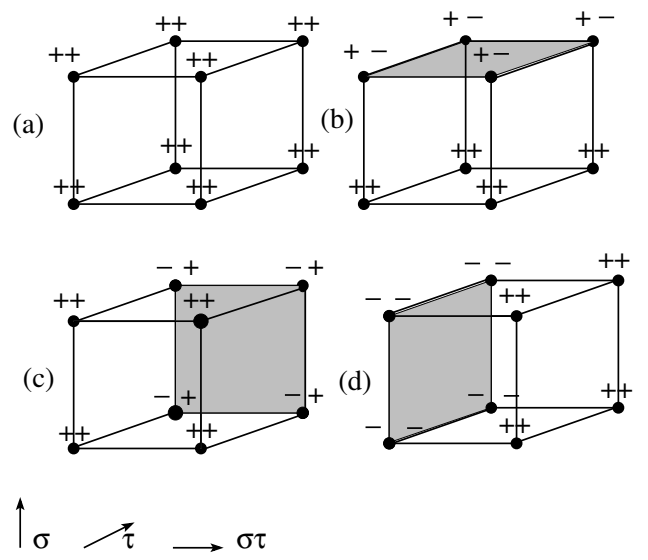

Fig. 3. Some possible ground-state spin configurations for the dual model on a cube with flipped spins. The $\sigma, \tau$ values are shown at each site, as are the directions of the anisotropic interactions between the spins. Once again, the entire lattice may be tiled by compatible combinations of such cubes.

\section{Standard first-order scaling}

We can obtain the basic finite-size scaling laws for first-order phase transitions by using a simple heuristic two-phase model, as described in $[55,56]$. In this, fluctuations within phases are ignored and we treat the phase transition as a sharp jump between the phases. A system thus spends a fraction $W_{\mathrm{o}}$ of the total time in a simulation in one of the $q$ ordered phases and a fraction $W_{\mathrm{d}}=1-W_{\mathrm{o}}$ in the disordered phase, with corresponding energies $\hat{e}_{\mathrm{o}}$ and $\hat{e}_{\mathrm{d}}$. In these expressions, the hat denotes quantities evaluated at the inverse transition temperature of the infinite system, $\beta^{\infty}$. The expectation values of the energy moments in the model are then given by weighted averages over the ordered and disordered phases

$$
\left\langle e^{n}\right\rangle=W_{\mathrm{o}} \hat{e}_{\mathrm{o}}^{n}+\left(1-W_{\mathrm{o}}\right) \hat{e}_{\mathrm{d}}^{n} .
$$

The specific heat $C_{V}(\beta, L)=-\beta^{2} \partial e(\beta, L) / \partial \beta$ in such an approximation is given by

$$
C_{V}(\beta, L)=L^{d} \beta^{2}\left(\left\langle e^{2}\right\rangle-\langle e\rangle^{2}\right)=L^{d} \beta^{2} W_{\mathrm{o}}\left(1-W_{\mathrm{o}}\right) \Delta \hat{e}^{2}
$$

where $\Delta \hat{e}=\hat{e}_{\mathrm{d}}-\hat{e}_{\mathrm{o}}$. Maximizing this with respect to $W_{\mathrm{o}}$ gives

$$
C_{V}^{\max }=L^{d}\left(\beta^{\infty} \Delta \hat{e} / 2\right)^{2}
$$

at the inverse temperature $\beta_{V}^{C_{V}^{\max }}(L)$ for which $W_{\mathrm{o}}=W_{\mathrm{d}}=0.5$. We can take the probability of being in any of the ordered states or the disordered state to be given by the standard Boltzmann factors

$$
p_{\mathrm{o}} \propto e^{-\beta L^{d} \hat{f}_{\mathrm{o}}} \text { and } p_{\mathrm{d}} \propto e^{-\beta L^{d} \hat{f}_{\mathrm{d}}},
$$

where $\hat{f}_{\mathrm{o}}, \hat{f}_{\mathrm{d}}$ are the free energy densities of the states. The fraction of time spent in the $q$ ordered states is proportional to $q p_{\mathrm{o}}$ and the fraction of time spent in the disordered state is proportional to $p_{\mathrm{d}}$, so the ratio of these is given by $W_{\mathrm{o}} / W_{\mathrm{d}} \simeq$ $q e^{-L^{d} \beta \hat{f}_{\mathrm{o}}} / e^{-\beta L^{d} \hat{f}_{\mathrm{d}}}$ up to exponentially small corrections in $L[16-19,57,58]$. Taking the logarithm of this ratio gives

$$
\ln \left(W_{\mathrm{o}} / W_{\mathrm{d}}\right) \simeq \ln q+L^{d} \beta\left(\hat{f}_{\mathrm{d}}-\hat{f}_{\mathrm{o}}\right) .
$$


Since $W_{\mathrm{o}}=W_{\mathrm{d}}$ at the finite-size specific-heat maximum, we find by expansion around $\beta^{\infty}$ that

$$
0=\ln q+L^{d} \Delta \hat{e}\left(\beta-\beta^{\infty}\right)+\ldots
$$

where $\Delta \hat{e}=\hat{e}_{\mathrm{d}}-\hat{e}_{\mathrm{o}}$, which can be solved for the location of finite-size peak of the specific heat:

$$
\beta_{V}^{C_{V}^{\max }}(L)=\beta^{\infty}-\frac{\ln q}{L^{d} \Delta \hat{e}}+\ldots
$$

Analogous calculations for the energetic Binder parameter

$$
B(\beta, L)=1-\frac{\left\langle e^{4}\right\rangle}{3\left\langle e^{2}\right\rangle^{2}}
$$

give $[55,56,59]$

$$
\beta^{B^{\mathrm{min}}}(L)=\beta^{\infty}-\frac{\ln \left(q \hat{e}_{\mathrm{o}}^{2} / \hat{e}_{\mathrm{d}}^{2}\right)}{L^{d} \Delta \hat{e}}+\ldots
$$

for the location $\beta^{B^{\mathrm{min}}}(L)$ of its minimum, which is an alternative estimator for the finite-size transition point.

The key features of finite-size scaling for first-order transitions are already exposed in this simple model. In Equation (6) we can see that the specific heat grows with the system volume $L^{d}$ and the finite-size values of the estimators for the (inverse) transition temperatures in Equation (10) and Equation (12) are shifted by $1 / L^{d}$. This behaviour is generic for finite-size scaling at first-order transitions for systems with periodic boundary conditions and it is confirmed by more sophisticated analyses using Pirogov-Sinai theory in cases where a contour expansion exists, such as for the $q$-state Potts model [16-19].

We can extend the discussion within the framework of the heuristic model to encompass non-periodic boundary conditions by allowing surface free-energy density terms, $\hat{s}_{\mathrm{O}}$ and $\hat{s}_{\mathrm{d}}$ for the ordered and disordered phases, respectively, in the Boltzmann factors. Note that $\hat{s}_{\mathrm{o}}$ and $\hat{s}_{\mathrm{d}}$ will in general not be equal since we have phase coexistence at the first-order transition point. With such boundary surface energy terms contributing, the Boltzmann factors become

$$
p_{\mathrm{o}} \propto e^{-\beta\left(L^{d} \hat{f}_{\mathrm{o}}+L^{d-1} \hat{s}_{\mathrm{o}}\right)} \text { and } p_{\mathrm{d}} \propto e^{-\beta\left(L^{d} \hat{f}_{\mathrm{d}}+L^{d-1} \hat{s}_{\mathrm{d}}\right)}
$$

and we can see that we might expect $1 / L$ leading corrections in such circumstances, which is again confirmed by more sophisticated analytical arguments and in simulations [60-63].

\section{Non-standard first-order scaling}

In principle the $3 d$ plaquette Ising model should provide an ideal arena for exploring the finer points of finite-size scaling at first-order transitions, since it has a relatively simple Hamiltonian and displays a strong first-order transition. However, high precision multicanonical simulations with periodic boundary conditions highlighted an anomaly: fits to the leading corrections to scaling for the two estimators of the transition point described in the previous section, $\beta^{C_{V}^{\max }}(L)$ and $\beta^{B^{\min }}(L)$, are much better if a leading $1 / L^{2}$ correction is assumed rather than the standard $1 / L^{3}[36]$. The goodness-of-fit parameter $Q$ for fits on the extremal locations of the specific heat, $\beta_{V}^{C_{V}^{\max }}$, and Binder's energy cumulant, $\beta^{B^{\min }}$, are shown in Figure 4. Similar behaviour is seen in the dual model of Equation (3). 

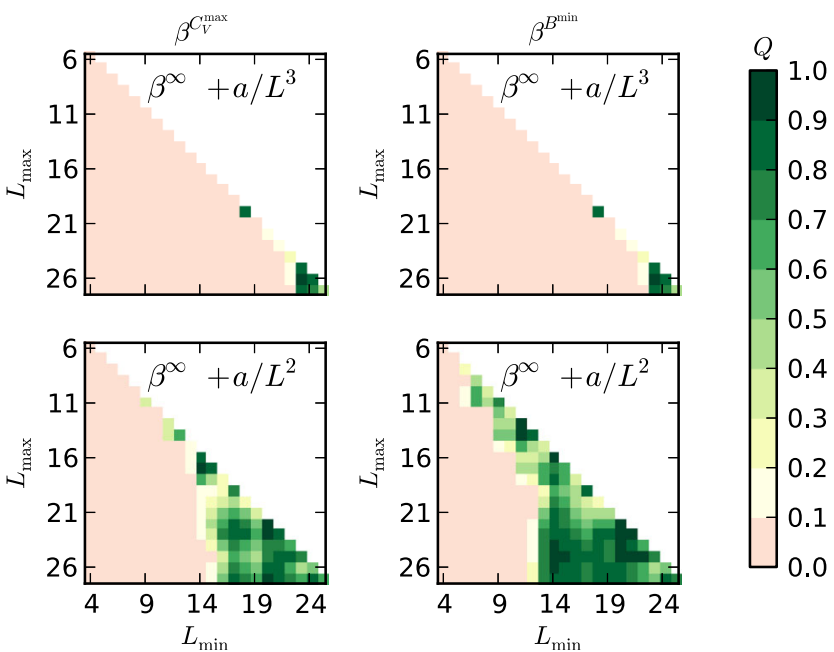

Fig. 4. Plot of the goodness-of-fit parameter $Q$ for fits on the extremal locations of the specific heat, $\beta_{V}^{C_{V}^{\max }}$, and Binder's energy cumulant, $\beta^{B^{\min }}$, of the $3 d$ plaquette Ising model for different fitting ranges $L_{\min }-L_{\max }$. Green regions indicate acceptable fits. Upper row: standard $1 / L^{3}$ finite-size scaling ansatz. Lower row: $1 / L^{2}$ finite-size scaling.

A resolution to the puzzle follows from noting that we have implicitly assumed that the degeneracy $q$ of the low-temperature phase is fixed, and in particular is independent of the system size in the calculations for the heuristic model in the preceding section. However, for the $3 d$ plaquette Ising model $q$ is size-dependent: $q=2^{3 L}$. It is also macroscopic, since it is increasing exponentially with the system size, although sub-extensive since it is increases as $\exp (L)$ rather than $\exp \left(L^{3}\right)$.

Taking $q=2^{3 L}$ we can rework the calculation of the leading scaling terms in Equations (10), (12) to take account of the size-dependence [36,37], giving

$$
\begin{aligned}
\beta_{V}^{C_{V}^{\max }}(L) & =\beta^{\infty}-\frac{\ln 2^{3 L}}{L^{3} \Delta \hat{e}}+\mathcal{O}\left(\left(\ln 2^{3 L}\right)^{2} L^{-6}\right) \\
& =\beta^{\infty}-\frac{3 \ln 2}{L^{2} \Delta \hat{e}}+\mathcal{O}\left(L^{-4}\right)
\end{aligned}
$$

and

$$
\begin{aligned}
\beta^{B^{\min }}(L) & =\beta^{\infty}-\frac{\ln \left(2^{3 L} \hat{e}_{\mathrm{o}}^{2} / \hat{e}_{\mathrm{d}}^{2}\right)}{L^{3} \Delta \hat{e}}+\mathcal{O}\left(\left(\ln \left(2^{3 L} \hat{e}_{\mathrm{o}}^{2} / \hat{e}_{\mathrm{d}}^{2}\right)\right)^{2} L^{-6}\right) \\
& =\beta^{\infty}-\frac{3 \ln 2}{L^{2} \Delta \hat{e}}-\frac{\ln \left(\hat{e}_{\mathrm{o}}^{2} / \hat{e}_{\mathrm{d}}^{2}\right)}{L^{3} \Delta \hat{e}}+\mathcal{O}\left(L^{-4}\right)
\end{aligned}
$$

for the estimators of the finite-size transition points. Both show that the leading contribution to the finite-size corrections is now $\propto L^{-2}$ rather than the customary $L^{-3}$ expected for periodic boundary conditions and size independent degeneracy. For the extremal values one finds

$$
C_{V}^{\max }(L)=L^{3}\left(\frac{\beta^{\infty} \Delta \hat{e}}{2}\right)^{2}+\mathcal{O}(L)
$$

and

$$
B^{\min }(L)=1-\frac{1}{12}\left(\frac{\hat{e}_{\mathrm{o}}}{\hat{e}_{\mathrm{d}}}+\frac{\hat{e}_{\mathrm{d}}}{\hat{e}_{\mathrm{o}}}\right)^{2}+\mathcal{O}\left(L^{-2}\right) .
$$


The inverse temperature where both peaks of the energy probability density have equal weight, $\beta^{\text {eqw }}(L)$, has a behaviour that, to leading order, coincides with the scaling of the location of the specific-heat maximum in equation (14),

$$
\beta^{\text {eqw }}(L)=\beta^{\infty}-\frac{3 \ln 2}{L^{2} \Delta \hat{e}}+\mathcal{O}\left(L^{-4}\right)
$$

The results of numerical simulations of the $3 d$ plaquette Ising model such as those shown in Figure 4 provide convincing verification of the non-standard $1 / L^{2}$ leading scaling corrections due to the macroscopic degeneracy.

\section{Simulational considerations}

Since in references $[36-39,41,42]$ we employed multicanonical simulations giving a flat energy distribution, reweighting techniques [64-67] can be used to get an estimator of the energy probability densities at different temperatures. $\beta^{\text {eqw }}$ is chosen systematically to minimise

$$
D^{\mathrm{eqw}}(\beta)=\left(\sum_{e<e_{\min }} p(e, \beta)-\sum_{e \geq e_{\min }} p(e, \beta)\right)^{2}
$$

where the energy of the minimum between the two peaks, $e_{\min }$, is determined beforehand to distinguish between the different phases. The location of the minimum, $\beta^{\text {eqw }}$, is then used to calculate the energy moments of the ordered and disordered phases,

$$
\begin{aligned}
& e_{\mathrm{o}}^{k}(L)=\sum_{e<e_{\min }} e^{k} p\left(e, \beta^{\mathrm{eqw}}\right) / \sum_{e<e_{\min }} p\left(e, \beta^{\mathrm{eqw}}\right), \\
& e_{\mathrm{d}}^{k}(L)=\sum_{e \geq e_{\min }} e^{k} p\left(e, \beta^{\mathrm{eqw}}\right) / \sum_{e \geq e_{\min }} p\left(e, \beta^{\mathrm{eqw}}\right),
\end{aligned}
$$

where $e_{\mathrm{o} / \mathrm{d}}(L)=e_{\mathrm{o} / \mathrm{d}}^{1}(L)$ is the energy in the respective phases, and their difference is an estimator of the latent heat $\Delta e(L)=e_{\mathrm{d}}(L)-e_{\mathrm{o}}(L)$. Also, the second and first moments combine to give the specific heat of the ordered and disordered phases,

$$
C_{\mathrm{o} / \mathrm{d}}(L)=\beta^{2} L^{d}\left(e_{\mathrm{o} / \mathrm{d}}^{2}(L)-\left(e_{\mathrm{o} / \mathrm{d}}(L)\right)^{2}\right) .
$$

To find the inverse transition temperature where both phases have equal height we minimise

$$
D^{\mathrm{eqh}}(\beta)=\left(\max _{e<e_{\min }}\{p(e, \beta)\}-\max _{e \geq e_{\min }}\{p(e, \beta)\}\right)^{2},
$$

as a function of $\beta$. The probability density $p\left(e, \beta^{\text {eqh }}\right)$ itself at $\beta^{\text {eqh }}$ is also of interest since one can make use of it to extract the reduced interface tension

$$
\sigma(L)=\frac{1}{2 L^{2}} \ln \left(\frac{\max \left\{p\left(e, \beta^{\mathrm{eqh}}\right)\right\}}{\min \left\{p\left(e, \beta^{\mathrm{eqh}}\right)\right\}}\right)
$$

for periodic boundary conditions.

If we collect the various estimates for physical quantities using the correct, modified leading $1 / L^{2}$ scaling corrections for periodic boundaries and $1 / L$ corrections for fixed boundaries we get consistent values across the original plaquette Hamiltonian with both fixed and periodic boundary conditions and the dual Hamiltonian with 
Table 1. Overview of resulting quantities of the infinite systems.

\begin{tabular}{|c|c|c|c|c|c|c|}
\hline Model & $\mathrm{bc}$ & $\beta^{\infty}$ & $\hat{e}_{\mathrm{o}}$ & $\hat{e}_{\mathrm{d}}$ & $\Delta \hat{e}$ & $\hat{\sigma}$ \\
\hline Original & Periodic & $0.551332(8)$ & $-1.468364(4)$ & $-0.617396(11)$ & $0.850968(18)$ & $0.12037(18)$ \\
\hline Dual & Periodic & $\begin{array}{c}0.55143(7) \\
{\left[\beta_{\text {dual }}^{\infty}=1.313\right.}\end{array}$ & $\begin{array}{l}-1.37644(21) \\
28(12)]\end{array}$ & $-0.88227(19)$ & $0.49402(26)$ & $0.1214(13)$ \\
\hline Original & Fixed & $0.55138(5)$ & $-1.4782(27)$ & $-0.790(4)$ & $0.694(4)$ & $0.0281(7)$ \\
\hline
\end{tabular}

periodic boundaries as shown in Table $1[36,37]$. We find an overall consistent value for the inverse transition temperature of

$$
\beta^{\infty}=0.551334(8) .
$$

We also find values for the interface tension of the original model and its dual with periodic boundary conditions of $\sigma=0.12037(18)$ and $\sigma=0.1214(13)$, respectively. The interface tension of the original model with fixed boundary conditions is found to be much smaller, $\sigma=0.0281(7)$.

\section{Order parameter(s)}

From Figure 1 it is clear that the standard magnetisation in the ordered lowtemperature phase will be zero, since there is no energy penalty for flipping the sign of a plane of spins and spin configurations in the low-temperature phase could contain arbitrary numbers of flipped planes of spins with respect to a purely ferromagnetic state. A possible alternative order parameter emerged from the work of Suzuki, who had investigated an anisotropic variant of the plaquette model many years ago [68]. He dubbed this the fuki-nuke ("no-ceiling" in Japanese) model, because the horizontal ceiling plaquettes have zero coupling. In the fuki-nuke model it was possible to define an (anisotropic) order parameter by using its hidden equivalence to a stack of standard $2 d$ Ising models. Suzuki and Hashizume returned to plaquette Ising models much more recently $[69,70]$ to observe that a similar order parameter to that used for the fuki-nuke model might still be viable for the isotropic $3 d$ plaquette Ising Hamiltonian, but in that case it should be independent of the orientation.

The anisotropic $3 d$ plaquette Hamiltonian for the fuki-nuke model is given by

$$
\begin{aligned}
H_{\text {fuki-nuke }}= & -J_{x} \sum_{x=1}^{L} \sum_{y=1}^{L} \sum_{z=1}^{L} \sigma_{x, y, z} \sigma_{x, y+1, z} \sigma_{x, y+1, z+1} \sigma_{x, y, z+1} \\
& -J_{y} \sum_{x=1}^{L} \sum_{y=1}^{L} \sum_{z=1}^{L} \sigma_{x, y, z} \sigma_{x+1, y, z} \sigma_{x+1, y, z+1} \sigma_{x, y, z+1}
\end{aligned}
$$

where we have now written the individual spin positions explicitly and set the coupling of the horizontal plaquettes $J_{z}=0$. This Hamiltonian may be rewritten as a stack of standard $2 d$ nearest-neighbour Ising models by defining bond spin variables $\tau_{x, y, z}=\sigma_{x, y, z} \sigma_{x, y, z+1}$ at each vertical lattice bond. With periodic (vertical) boundary conditions we must impose the constraints $\prod_{z=1}^{L} \tau_{x, y, z}=1$ which preclude an explicit solution [71], but in the case of free boundary conditions the Ising layers completely decouple, with the Hamiltonian

$$
H_{\text {fuki-nuke }}=-\sum_{x=1}^{L} \sum_{y=1}^{L} \sum_{z=1}^{L}\left(\tau_{x, y, z} \tau_{x+1, y, z}+\tau_{x, y, z} \tau_{x, y+1, z}\right),
$$


where we have set $J_{x}=J_{y}=1$ for simplicity. The partition function for free boundary conditions is

$$
\begin{aligned}
Z_{\text {fuki-nuke }} & =\sum_{\{\tau\}} \exp \left(-\beta H_{\text {fuki-nuke }}(\{\tau\})\right) \\
& =2^{L^{2}} \sum_{\left\{\tau_{x, y, z \neq L}\right\}} \prod_{z=1}^{L-1} \exp \left(\beta \sum_{x=1}^{L} \sum_{y=1}^{L}\left(\tau_{x, y, z} \tau_{x+1, y, z}+\tau_{x, y, z} \tau_{x, y+1, z}\right)\right) \\
& =2^{L^{2}} \prod_{z=1}^{L-1} \sum_{\left\{\tau_{x, y}\right\}_{z}} \exp \left(\beta \sum_{x=1}^{L} \sum_{y=1}^{L}\left(\tau_{x, y, z} \tau_{x+1, y, z}+\tau_{x, y, z} \tau_{x, y+1, z}\right)\right) \\
& =2^{L^{2}} \prod_{z=1}^{L-1} Z_{2 d \text { Ising }}=2^{L^{2}}\left(Z_{2 d \text { Ising }}\right)^{L-1},
\end{aligned}
$$

where $\left\{\tau_{x, y}\right\}_{z}$ denotes summation over all $\tau$-spins with a given $z$-component and

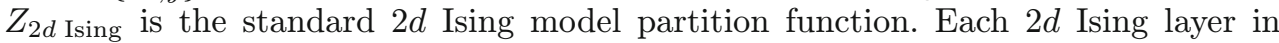
Equation (27) magnetises independently at the $2 d$ Ising model transition temperature. For the case of periodic boundaries we would expect the same thermodynamic behaviour with only the corrections to scaling being affected by the constraints arising from the boundary conditions.

When the model is expressed in terms of the $\tau$ spins, a suitable order parameter for a single Ising layer is the standard magnetisation

$$
m_{2 d, z}=\left\langle\frac{1}{L^{2}} \sum_{x=1}^{L} \sum_{y=1}^{L} \tau_{x, y, z}\right\rangle
$$

which when translated back to the original $\sigma$ spins gives

$$
m_{2 d, z}=\left\langle\frac{1}{L^{2}} \sum_{x=1}^{L} \sum_{y=1}^{L} \sigma_{x, y, z} \sigma_{x, y, z+1}\right\rangle .
$$

This single layer nearest (vertical) neighbour correlator in the fuki-nuke model will thus behave like the standard $2 d$ Ising magnetisation $\pm\left|\beta-\beta_{c}\right|^{\frac{1}{8}}$ near the critical point $\beta_{c}=\frac{1}{2} \ln (1+\sqrt{2})$.

There are two obvious possibilities for constructing a pseudo-3d order parameter from the layer magnetisations in the fuki-nuke model, both chosen to eliminate cancellations between differently magnetised planes of Ising spins [40]. The first is to take the absolute value of the magnetisation in each plane

$$
m_{\mathrm{abs}}=\left\langle\frac{1}{L^{3}} \sum_{z=1}^{L}\left|\sum_{x=1}^{L} \sum_{y=1}^{L} \sigma_{x, y, z} \sigma_{x, y, z+1}\right|\right\rangle,
$$

and the second is to square the magnetisation of each plane,

$$
m_{\mathrm{sq}}=\left\langle\frac{1}{L^{5}} \sum_{z=1}^{L}\left(\sum_{x=1}^{L} \sum_{y=1}^{L} \sigma_{x, y, z} \sigma_{x, y, z+1}\right)^{2}\right\rangle .
$$

We have retained the various normalizing factors in Equations (30) and (31) for a cubic lattice with $L^{3}$ sites. 
Suzuki's and Hashizume's suggestion $[69,70]$ was that we could define the order parameter for the isotropically coupled plaquette model in a similar fashion by using the same nearest-neighbour correlator in any of the three directions, e.g.,

$$
m_{\mathrm{abs}}^{x}=\left\langle\frac{1}{L^{3}} \sum_{x=1}^{L}\left|\sum_{y=1}^{L} \sum_{z=1}^{L} \sigma_{x, y, z} \sigma_{x+1, y, z}\right|\right\rangle,
$$

with the obvious analogous definition for the $y, z$ directions and where we have again assumed periodic boundary conditions. The squared magnetisations could be defined in a similar manner as

$$
m_{\mathrm{sq}}^{x}=\left\langle\frac{1}{L^{5}} \sum_{x=1}^{L}\left(\sum_{y=1}^{L} \sum_{z=1}^{L} \sigma_{x, y, z} \sigma_{x+1, y, z}\right)^{2}\right\rangle
$$

and similarly for the $y, z$ directions. In the isotropic case we would expect $m_{\text {abs }}^{x}=$ $m_{\mathrm{abs}}^{y}=m_{\mathrm{abs}}^{z}$ and similarly for the squared quantities. Lipowski had previously suggested [6] using an order parameter akin to $m_{\mathrm{sq}}$ in equation (31).

If we consider the susceptibilities $\chi$ for the various order-parameter candidates, the peak locations $\beta^{\chi}(L)$ for the different lattice sizes $L$ can be fitted using the modified first-order scaling laws discussed in the preceding section,

$$
\beta^{\chi}(L)=\beta^{\infty}+a / L^{2}+b / L^{3} .
$$

This gives for the estimate of the inverse critical temperature $\beta^{\chi}(L)$ from the fuki-nuke susceptibility $\chi_{m_{\text {abs }}^{x}}$

$$
\beta^{\chi_{m_{\mathrm{abs}}^{x}}}(L)=0.55137(3)-2.46(3) / L^{2}+2.4(3) / L^{3},
$$

with a goodness-of-fit parameter $Q=0.64$ and 12 degrees of freedom left as shown in Figure 5. Fits to the other directions $m_{\text {abs }}^{y, z}$ and fits to the peak location of the susceptibilities of $m_{\mathrm{sq}}^{x, y, z}$ give the same parameters within error bars and are of comparable quality. The estimates of the transition point obtained from the various $m_{\mathrm{abs}}$ and $m_{\mathrm{sq}}$ are thus independent of the directions $x, y, z$, confirming Suzuki's and Hashizume's hypothesis, and their values are consistent with the estimates in Table 1 and Equation (24) obtained from $\beta^{C^{\max }}(L)$ and $\beta^{B^{\min }}(L)[41,42]$.

We can see that the low-temperature degeneracy has impacted both the definition of the magnetic order parameter, giving rise to an order parameter that is a hybrid $2 d / 3 d$ construct and the finite-size scaling of the associated susceptibility, which also shows the leading $1 / L^{2}$ corrections seen in energetic quantities such as the specificheat peak.

\section{A curious symmetry - quantum aspects: duality and fractons}

A further consequence of the planar flip symmetry is found in a Hamiltonian related to the quantum dual of the plaquette model. This fits into the general framework developed in $[43,44]$ in which novel fracton topological phases are constructed by gauging symmetries acting on subsystems of dimension $2 \leq d_{s}<d$. Since the spin-flip symmetry in the $3 d$ plaquette model acts on $2 d$ planes it has precisely this property. The procedure for constructing the fracton Hamiltonian follows closely that of the Kitaev toric code, giving commuting electric and magnetic operators. 

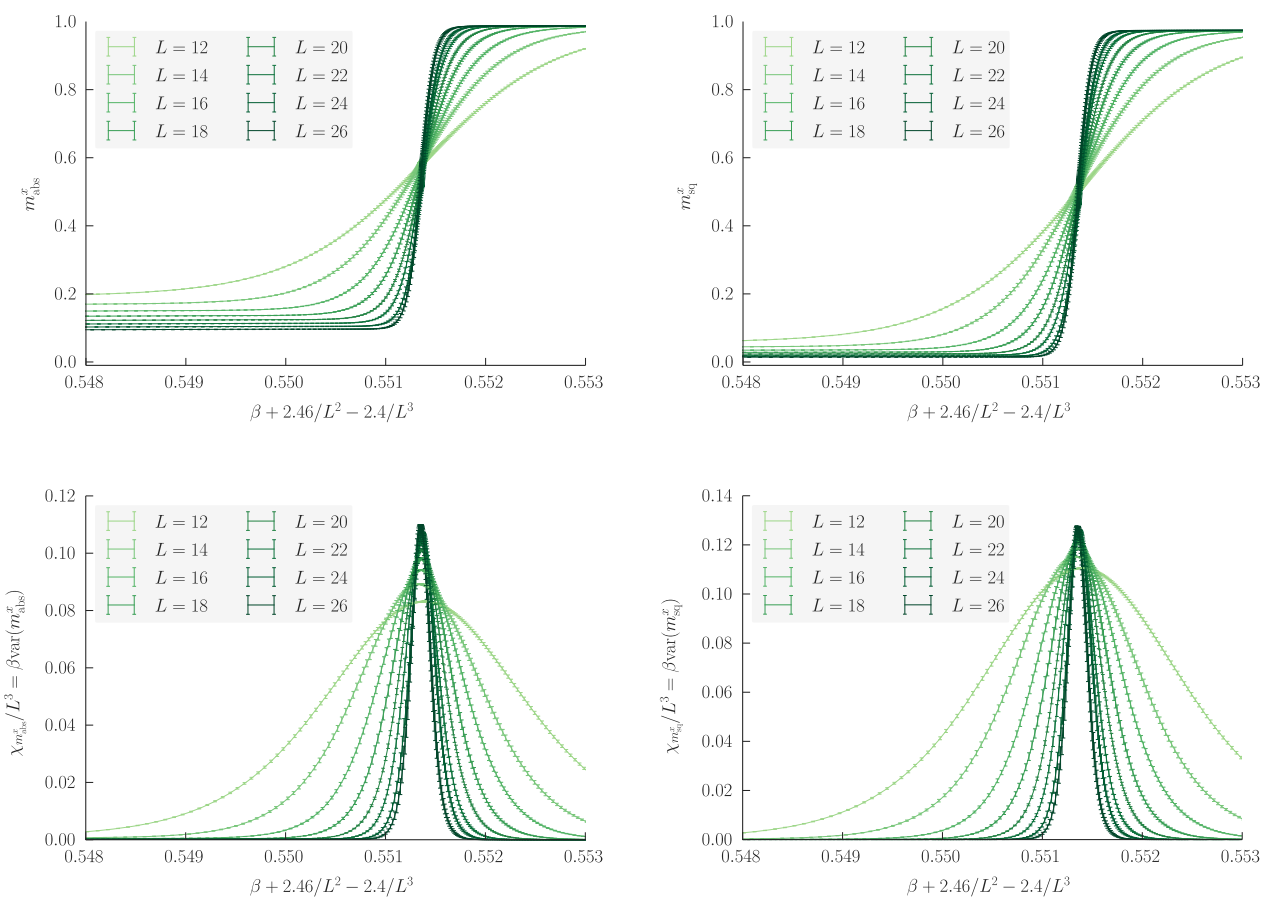

Fig. 5. The fuki-nuke parameters $m_{\mathrm{abs}}^{x}$ and $m_{\mathrm{sq}}^{x}$ (upper row) along with their respective susceptibilities $\chi$ normalised by the system volume (lower row) over shifted inverse temperature $\beta$ for several lattice sizes $L$.

The quantum spin version of the plaquette model promotes the classical Ising spins $\sigma$ to operators, represented by Pauli matrices $\sigma^{z}$ and spin flips are implemented with $\sigma^{x},{ }^{1}$ so an external transverse field term may be added to the Hamiltonian to give

$$
H_{0}=-t \sum_{[i, j, k, l]} \sigma_{i}^{z} \sigma_{j}^{z} \sigma_{k}^{z} \sigma_{l}^{z}-h \sum_{i} \sigma_{i}^{x}
$$

The quantum dual of this Hamiltonian is given by

$$
H_{\text {nexus }}=-t \sum_{i} \tau_{i}^{z}-h \sum_{i} A_{i}
$$

where the nexus spin operators $\tau$ live at the centre of each plaquette on the original lattice, i.e., the centre of links on the dual lattice. They play the same role as the link gauge spins introduced in dualising the standard $\mathbb{Z}_{2}$ Ising model in $3 d$. This generalised gauging/dualising procedure may be applied to other classical systems with a subsystem symmetry and leads, inter alia, to the Haah code model [45-48], as well as other fracton models.

\footnotetext{
${ }^{1}$ At the risk of causing confusion we have retained our nomenclature $\sigma$ for the original spins and called the dual nexus spins $\tau$, which is the opposite of the convention employed in [43].
} 


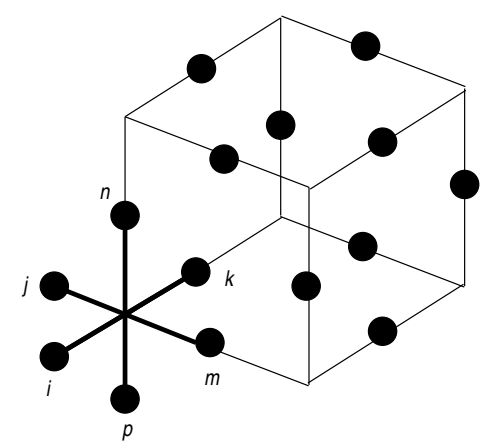

Fig. 6. The spins involved in defining the fracton Hamiltonian, $H_{\text {fracton }}$, shown on the edges of a dual lattice cube (for $A_{i}$ terms) and on a "star" at one corner (for $B_{i}$ terms).

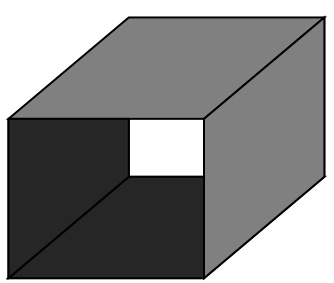

Fig. 7. One of the three possible orientations of a product of four plaquettes round a cube.

With the nexus spins in $H_{\text {nexus }}$ residing at the centres of the links of the dual cubic lattice, the nexus charge operator $A_{i}$ is given by

$$
A_{i} \equiv \prod_{j \in P(i)} \tau_{j}^{x},
$$

where the set $P(i)$ specifies the locations of multi-spin interactions that are affected by a spin flip using $\sigma_{i}^{x}$ in $H_{0}$. For the plaquette model $P(i)$ comprises the twelve $\tau$ spins on the edges of a cube in the dual lattice as shown in Figure 6, coming from the twelve plaquettes that are affected by acting with a single spin-flip operator $\sigma_{i}^{x}$ in $H_{0}$, which lies at the centre of the dual lattice cube.

We also need to impose constraints on the physical states in the theory that arise from rewriting $H_{0}$ in terms of the nexus spins, $\tau$, as is apparent already at the classical level. If we consider the product of four plaquettes around a cube shown in Figure 7 we can see that this must be one, and similarly for the other orientations. This means that products of the nexus spins associated with the shaded plaquettes must also be one and translates into constraints on the physical states in the quantum spin version of the theory,

$$
B_{i}|\Psi\rangle=|\Psi\rangle
$$

where the $B_{i}$ are the three star products of the nexus spin operators

$$
\begin{aligned}
& B_{i}^{(x z)}=\tau_{i}^{z} \tau_{p}^{z} \tau_{n}^{z} \tau_{k}^{z}, \\
& B_{i}^{(x y)}=\tau_{i}^{z} \tau_{j}^{z} \tau_{k}^{z} \tau_{m}^{z}, \\
& B_{i}^{(y z)}=\tau_{j}^{z} \tau_{n}^{z} \tau_{m}^{z} \tau_{p}^{z}
\end{aligned}
$$

and can also be thought of as generalised monopole charge operators. These are also shown in Figure 6. The effect of the constraints is to restrict the Hilbert space of the 
$\{\tau\}$ to be the domain-wall configurations of the ordered phase of $H_{0}$. It is interesting to note that a different approach in which dual spins are placed at the centre of the cubes wrapped by the shaded plaquettes, rather than at the centre of each plaquette, leads to the Ashkin-Teller-like Hamiltonian of Equation (3).

The X-Cube fracton Hamiltonian

$$
H_{\text {fracton }}=-\sum_{i} B_{i}-\sum_{i} A_{i}
$$

named after the configuration of the interaction terms seen in Figure 6, was argued in [43] to display fracton topological order with two distinguishing characteristics: it had a sub-extensive topological degeneracy and the motion of both electric and magnetic excitations was constrained.

The flip symmetry for planes of spins plays a central role in both these deductions. In the original plaquette Hamiltonian $H_{0}$ the symmetry was generated by products of $\sigma_{i}^{x}$ in the plane. As we have seen, the associated ground states in the classical system have a sub-extensive $2^{3 L}$ degeneracy, which remains the case in the quantum system when $t / h \gg 1$. The dual representation of the planar product of $\sigma_{i}^{x}$ 's is a product of the nexus charges over the same plane and the plaquette interaction terms in $H_{0}$ are dual to individual nexus spins $\tau_{i}^{z}$. The dual relation to the spin flip commuting with the plaquette interaction term is thus

$$
\left[\tau_{i}^{z}, \prod_{i \in \Sigma} A_{i}\right]=0
$$

where $\Sigma$ is a planar region along which spins are flipped. This can only be satisfied if $\prod_{i \in \Sigma} A_{i}=1$ on such a plane, so each plane provides one constraint for the nexus charges.

In general with $N$ nexus spins $\tau$ and $M$ nexus charges $A_{i}$ and monopole operators $B_{i}$ in a theory, the topological ground-state degeneracy on a torus (i.e., a lattice with periodic boundary conditions) would be $D=2^{k+M-N}$ where $k$ is the number of constraints satisfied by the $A_{i}$ and $B_{i}$. For the fracton Hamiltonian $M=N$ and the number of constraints is $k=3 L$, one from each plane, from the arguments above. The sub-extensive ground-state degeneracy due to the flip symmetry of the plaquette model thus translates into the sub-extensive topological degeneracy of the fracton Hamiltonian.

A second consequence of the symmetry is that the excitations in the X-Cube model have restricted mobility. The location of electric excitations created in the ground state of $H_{\text {fracton }}$ by a product of $\tau_{i}^{z}$ operators on some planar subset $\Sigma$ of the lattice

$$
W=\prod_{i \in \Sigma} \tau_{i}^{z}
$$

is identical to the location of the planar spin flips created by the dual operator

$$
\tilde{W}=\prod_{\square_{i}} \sigma^{z} \sigma^{z} \sigma^{z} \sigma^{z}
$$

acting on the paramagnetic state of $H_{0}$, where the product is over the plaquettes $\square_{i}$ associated with the nexus spins $\tau_{i}^{z}$ in the same region $\Sigma$.

Since $\tilde{W}$ cannot create an isolated pair of spin-flip excitations in the paramagnetic state, $W$ cannot create an isolated pair of fracton excitations and four will appear at the corners of a rectangular region of flipped spins. This is characteristic of so-called type I fracton order [43]. Although the fracton (electric) excitations are pinned in this 
model, a Wilson line of $\tau^{x}$ operators acting on the fracton Hamiltonian ground state generates a pair of magnetic excitations (which come in two flavours) at the ends of the line which can continue to move along the line. Changing direction generates a further magnetic excitation of a different flavour at the corner.

\section{Conclusions}

The $3 d$ plaquette Ising model possesses an unusual planar spin-flip symmetry and macroscopic (but sub-extensive) low-temperature phase degeneracy. This has consequences for the finite-size scaling at its first-order transition, which displays $1 / L^{2}$ leading corrections, and for the definition of the magnetic order parameter, which has a hybrid $2 d / 3 d$ form. We described the symmetry in the plaquette model and its dual and outlined the necessary adaptions to standard first-order finite-size scaling in order to take account of macroscopic degeneracy. The anisotropic, fuki-nuke, variant of the plaquette model was then used to deduce a suitable order parameter.

Finally, we looked at implications of the symmetry in the quantum version of the model. Here, a Hamiltonian related to the quantum dual of the plaquette model describes fracton topological phases, which have point-like topological excitations that appear at the corners of membrane-like operators, such as planar regions of a $3 d$ lattice. This fracton Hamiltonian displays a macroscopic, sub-extensive topological degeneracy that is directly related to the spin-flip symmetry of the plaquette model, as is the restricted mobility of the topological excitations in the model.

Further numerical exploration of models with subsystem symmetries analogous to the plaquette model might prove profitable for the understanding of exotic topological phases of matter, such as the fracton phase discussed here. For instance, Monte Carlo simulations of the $\left(3 d\right.$, classical) $\mathbb{Z}_{2}$ gauge-Higgs model were employed to good effect in investigating the phase diagram of the (2d, quantum) toric code by using the $d$-dimensional quantum to $d+1$-dimensional classical mapping [72]

We would like to thank the organizers of the PTCP conference. This work was supported by the Deutsche Forschungsgemeinschaft (DFG) through the Collaborative Research Centre SFB/TRR 102 (project B04), the Deutsch-Französische Hochschule (DFH-UFA) through the Doctoral College " $\mathbb{L}^{4}$ " under Grant No. CDFA-02-07, and by the EU Marie Curie IRSES Network DIONICOS under Contract No. PIRSES-GA-2013-612 707.

\section{References}

1. E. Ising, Z. Phys. 31, 253 (1925)

2. B.M. McCoy, T.T. Wu, The Two-Dimensional Ising Model (Harvard University Press, Cambridge, 1973)

3. L. Onsager, Phys. Rev. 65, 117 (1944)

4. B. Kaufman, Phys. Rev. 76, 1232 (1949)

5. A.E. Ferdinand, M.E. Fisher, Phys. Rev. 185, 832 (1969)

6. A. Lipowski, J. Phys. A: Math. Gen. 30, 7365 (1997)

7. D.A. Johnston, A. Lipowski, R.P.K.C. Malmini, in Rugged Free Energy Landscapes: Common Computational Approaches to Spin Glasses, Structural Glasses and Biological Macromolecules, edited by W. Janke, Lecture Notes in Physics (Springer, Berlin, 2008), Vol. 736, p. 173

8. Y. Imry, Phys. Rev. B 21, 2042 (1980)

9. K. Binder, Z. Phys. B 43, 119 (1981)

10. M.E. Fisher, A.N. Berker, Phys. Rev. B 26, 2507 (1982)

11. V. Privman, M.E. Fisher, J. Stat. Phys. 33, 385 (1983) 
12. K. Binder, D.P. Landau, Phys. Rev. B 30, 1477 (1984)

13. M.S.S. Challa, D.P. Landau, K. Binder, Phys. Rev. B 34, 1841 (1986)

14. P. Peczak, D.P. Landau, Phys. Rev. B 39, 11932 (1989)

15. V. Privman, J. Rudnik, J. Stat. Phys. 60, 551 (1990)

16. C. Borgs, R. Kotecký, J. Stat. Phys. 61, 79 (1990)

17. C. Borgs, R. Kotecký, S. Miracle-Solé, J. Stat. Phys. 62, 529 (1991)

18. C. Borgs, R. Kotecký, Phys. Rev. Lett. 68, 1734 (1992)

19. C. Borgs, J.Z. Imbrie, J. Stat. Phys. 69, 487 (1992)

20. T.R. Kirkpatrick, D. Thirumalai, Phys. Rev. Lett. 58, 2091 (1987)

21. T.R. Kirkpatrick, D. Thirumalai, Phys. Rev. B 36, 5388 (1987)

22. T.R. Kirkpatrick, D. Thirumalai, Rev. Mod. Phys. 87, 183 (2015)

23. R.V. Ambartzumian, G.K. Savvidy, K.G. Savvidy, G.S. Sukiasian, Phys. Lett. B 275, $99(1992)$

24. G.K. Savvidy, K.G. Savvidy, Mod. Phys. Lett. A 08, 2963 (1993)

25. G.K. Savvidy, K.G. Savvidy, Int. J. Mod. Phys. A 08, 3993 (1993)

26. G.K. Savvidy, F.J. Wegner, Nucl. Phys. B 413, 605 (1994)

27. G.K. Savvidy, K.G. Savvidy, Phys. Lett. B 324, 72 (1994)

28. G.K. Bathas, E. Floratos, G.K. Savvidy, K.G. Savvidy, Mod. Phys. Lett. A 10, 2695 (1995)

29. G.K. Savvidy, K.G. Savvidy, P.G. Savvidy, Phys. Lett. A 221, 233 (1996)

30. G.K. Savvidy, J. High Energy Phys. 9, 44 (2000)

31. G.K. Savvidy, Mod. Phys. Lett. B 29, 1550203 (2015)

32. A. Cappi, P. Colangelo, G. Gonella, A. Maritan, Nucl. Phys. B 370, 659 (1992)

33. E.N.M. Cirillo, G. Gonnella, D.A. Johnston, A. Pelizzola, Phys. Lett. A 226, 59 (1997)

34. E.N.M. Cirillo, G. Gonnella, A. Pelizzola, Phys. Rev. E 55, R17 (1997)

35. D. Espriu, M. Baig, D.A. Johnston, R.P.K.C. Malmini, J. Phys. A: Math. Gen. 30, 405 (1997)

36. M. Mueller, W. Janke, D.A. Johnston, Phys. Rev. Lett. 112, 200601 (2014)

37. M. Mueller, D.A. Johnston, W. Janke, Nucl. Phys. B 888, 214 (2014)

38. M. Mueller, W. Janke, D.A. Johnston, Physics Procedia 57, 68 (2014)

39. W. Janke, M. Mueller, D.A. Johnston, J. Phys. Conf. Ser. 640, 012002 (2015)

40. D.A. Johnston, J. Phys. A: Math. Theor. 45, 405001 (2012)

41. M. Mueller, D.A. Johnston, W. Janke, Nucl. Phys. B 894, 1 (2015)

42. D.A. Johnston, M. Mueller, W. Janke, Mod. Phys. Lett. B 29, 1550109 (2015)

43. S. Vijay, J. Haah, L. Fu, Phys. Rev. B 94, 235157 (2016)

44. D.J. Williamson, Phys. Rev. B 94, 155128 (2016)

45. C. Chamon, Phys. Rev. Lett. 94, 040402 (2005)

46. S. Bravyi, B. Leemhuis, B. Terhal, Ann. Phys. 326, 839 (2011)

47. J. Haah, Phys. Rev. A 83, 042330 (2011)

48. B. Yoshida, Phys. Rev. B 88, 125122 (2013)

49. D.A. Johnston, R.P.K.C. Malmini, Phys. Lett. B 378, 87 (1996)

50. R. Pietig, F. Wegner, Nucl. Phys. B 466, 513 (1996)

51. R. Pietig, F. Wegner, Nucl. Phys. B 525, 549 (1998)

52. J. Slawny, J. Stat. Phys. 20, 711 (1979)

53. A.D. Beath, D.H. Ryan, Phys. Rev. B 73, 174416 (2006)

54. D.A. Johnston, R.P.K.C.M. Ranasinghe, J. Phys. A: Math. Theor. 44, 295004 (2011)

55. W. Janke, Phys. Rev. B 47, 14757 (1993)

56. W. Janke, in Computer Simulations of Surfaces and Interfaces, edited by B. Dünweg, D.P. Landau, A.I. Milchev, NATO Science Series, II. Math. Phys. Chem. (Kluwer, Dordrecht, 2003), Vol. 114, p. 111

57. C. Borgs, W. Janke, Phys. Rev. Lett. 68, 1738 (1992)

58. W. Janke, R. Villanova, Nucl. Phys. B 489 [FS], 679 (1997)

59. J. Lee, J.M. Kosterlitz, Phys. Rev. B 43, 3265 (1991)

60. C. Borgs, R. Kotecký, J. Stat. Phys. 79, 43 (1995)

61. R. Kotecký, I. Medved, J. Stat. Phys. 104, 905 (2001) 
62. C. Borgs, R. Kotecký, I. Medved, J. Stat. Phys. 109, 67 (2002)

63. M. Baig, R. Villanova, Phys. Rev. B 65, 094428 (2002)

64. A.M. Ferrenberg, R.H. Swendsen, Phys. Rev. Lett. 61, 2635 (1988)

65. W. Janke, in Computational Physics: Selected Methods - Simple Exercises - Serious Applications, edited by K.H. Hoffmann, M. Schreiber (Springer, Berlin, 1996), p. 10

66. W. Janke, Physica A 254, 164 (1998)

67. W. Janke, in Computer Simulations of Surfaces and Interfaces, edited by B. Dünweg, D.P. Landau, A.I. Milchev, NATO Science Series, II. Math. Phys. Chem. (Kluwer, Dordrecht, 2003), Vol. 114, p. 137

68. M. Suzuki, Phys. Rev. Lett. 28, 507 (1972)

69. Y. Hashizume, M. Suzuki, Int. J. Mod. Phys. B 25, 73 (2011)

70. Y. Hashizume, M. Suzuki, Int. J. Mod. Phys. B 25, 3529 (2011)

71. M. Mueller, D.A. Johnston, W. Janke, Nucl. Phys. B 914, 388 (2017)

72. I.S. Tupitsyn, A. Kitaev, N.V. Prokof'ev, P.C.E. Stamp, Phys. Rev. B 82, 085114 (2010)

Open Access This is an Open Access article distributed under the terms of the Creative Commons Attribution License (http://creativecommons.org/licenses/by/4.0), which permits unrestricted use, distribution, and reproduction in any medium, provided the original work is properly cited. 